\title{
Designing of an innovative extrusion system for metallic parts made by desktop 3D printing method
}

\author{
Razvan Pacurar*, Ancuta Pacurar and Serban Pop \\ Technical University of Cluj-Napoca, Faculty of Machine Building, Department of Manufacturing \\ Engineering, B-dul Muncii no. 103-105, 400641, Cluj-Napoca, Romania
}

\begin{abstract}
Desktop 3D Printing for metallic parts is a new direction and represents one of the latest trends within the Additive Manufacturing domain, being given and defined mainly by the requirements of the industrial companies in terms of materials and products that needs to be rapidly delivered on the market. The article presents an innovative solution that was developed at the Technical University of Cluj-Napoca in this domain, for the Rapid Product Development of Parts made by metal extrusion.
\end{abstract}

\section{Introduction}

Nowadays, 3D printing is more and more widespread in various fields, such as automotive, medicine, aeronautics, etc. [1-3]. This technological process offers a number of advantages over conventional manufacturing technologies, especially regarding to the possibility of producing complex models with high productivity and the use of cheap materials for manufacturing $[4,5]$. One of the main disadvantages of the $3 \mathrm{D}$ printing technologies is the limited range of materials, with most of the 3D Printers only being used for the realization of parts made from plastic materials [6]. Possibility of using metallic materials for producing parts with the 3D desktop printing method (e.g. Fused Deposition Modeling) has been considered by different researchers in the world [7, 8]. For example, at the University of Texas at El Paso, USA, a FDM 3000 system was modified in order to achieve controlled deposition of eutectic Bi58Sn42 and non-eutectic Sn60Bi40 materials, which are metallic alloys with very low melting temperature [9]. The made research were focused on the redesign of the extruded system of the 3D printer, in such way to be able to produce metallic parts for applications in electronics. Technological parameters were optimized to manufacture samples that could be further on mechanically and electrically tested. Interfacial phenomena between layers for such structures were also analyzed with optical microscopes, proving the fact that such method is reliable to produce metallic components by using the desktop 3D printing method. Interesting and similar research leading to the same conclusion has been reported also by a group of researchers from the University of Rostock in Germany [10]. In this case a very interesting combination between thermoplastic extrusion and metallic

\footnotetext{
*Corresponding author: razvan.pacurar@tcm.utcluj.ro
} 
part manufacturing by Metal Injection Moulding method in an original additive extrusion designed system has been presented. Extruding parameters were very well optimized, so as the metallic parts were manufactured with high tolerances at the end. The shrinkage along $\mathrm{z}$ direction had an average value of $15.8 \%$, slightly higher than the shrinkage along $x-y$ direction with an average value of $14.49 \%$. The reached density of the metallic parts produced by using the original designed and realized extruding system was closed to $96 \%$. Starting from the state of the art in the field and encouraged by the results of the research obtained so far, the possibility of designing and manufacturing of a sub-assembly extrusion system (originally conceived) required for the realization of metallic parts made by desktop $3 \mathrm{D}$ printing method at the Technical University of Cluj-Napoca (TUCN) has been considered.

\section{Designing of the extrusion system}

Three constructive versions were proposed based on the principle used by the classic 3D printers, the extruded material being in the form of a filament. This type of extruder incorporates a stepper motor, which drives a filament feeding mechanism, generally consisting of a driving wheel, actuated by the stepper motor and a driven wheel, the filament being fed through the respective wheels (see Figure1).

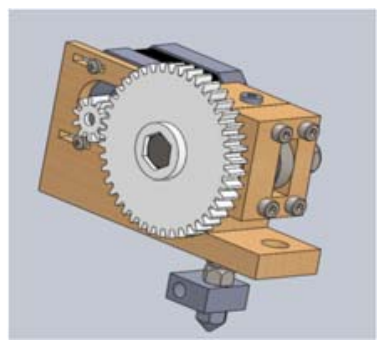

a

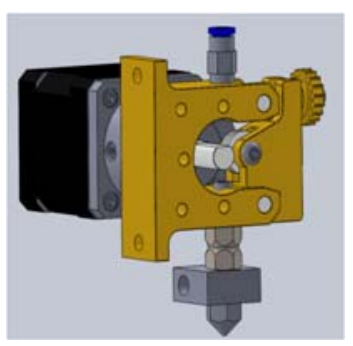

b

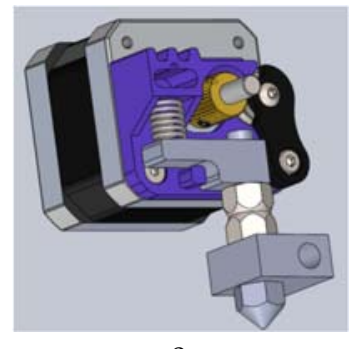

$\mathrm{C}$

Fig. 1. Constructive versions of the 3D sub-assembly extrusion systems.

The construction of the first type of extruder shown in Figure1 a) is quite complex, due to the fact that it has a wooden casing with complex geometry and two bearings incorporated into the casing. Also, this version has two spur gears, which are also complex parts. The heating of the hot-end is run by induction by means of an electrical resistance, which is mounted inside the crucible and commanded by the printer's power electronics floor. Even though this is an efficient version, its complexity is high, so the manufacturing price for the extruder will be high, as compared to the other versions.

The construction of the $2^{\text {nd }}$ version of the extruder presented in Figure $1 \mathrm{~b}$ ) is quite simple, the wire being fed directly by the shaft of the stepper motor. The filament feeding mechanism consists of two metallic components, which sustain the entire sub-assembly and through them the extrusion sub-assembly will be fixed on the 3D printer. This type of extruder also has a driven wheel, which is put in motion by the shaft of the motor and the wire, when it passes between them. The clearance between the shaft and the wheel can be adjusted from the wheel in the left of the assembly. The hot-end design is the same as the one presented in the case of constructive version 1 presented in Figure 1 a) and it is also heated by means of an electrical resistance, which is mounted inside the crucible. Even though it is a simple version, the feeding of the filament was not considered as being a very good solution, because the grip between the shaft and the driven wheel is not very good, so the feeding force provided by this version might not be high enough for the filament to pass through the nozzle at the required rate. 
The constructive version 3 presented in Figure $1 \mathrm{c}$ ) is very similar to the one presented in Figure $1 \mathrm{~b}$ ), because it is based on the same wire feeding mechanism and it also has very small overall dimensions, even smaller than version presented in Fig 1 b), so it could be fitted on any desktop 3D printer. By being smaller, it also has a lower mass, which means that the stress on the motors, guides and columns will be smaller, so the durability of the $3 \mathrm{D}$ printer will be higher in this case. The hot-end is also the same as the one presented in the previous versions, having the same components and being heated by an electrical resistance. This version of the sub-assembly extrusion system presented in Fig $1 \mathrm{c}$ ) has better gripping capabilities than the previous ones, because it has a brass wheel, having small teeth attached on the shaft of the stepper motor, so the wire won't slip between the brass wheel and the driven wheel. In the case of this sub-assembly extrusion system, there is no need to manually adjust the clearance between the two wheels, because it has rigid automation by means of a spring.

\section{Comparative analyses of the constructive versions and final validation of the selected version}

In order to choose which version is optimum from the point of view of the overall dimensions, total mass of the assembly, wire feeding accuracy, reliability and maintenance and the total manufacturing costs, a comparative analysis of the constructive versions was made as presented in Table 1.

Table 1. Comparative analyses of the constructive versions.

\begin{tabular}{|c|c|c|c|}
\hline & Version I & Version II & Version III \\
\hline Overall dimensions [mm] & $113 \times 60$ & $92 \times 69$ & $75 \times 42$ \\
\hline Total mass [g] & 603 & 458 & 384 \\
\hline Wire feeding accuracy & High & Low & High \\
\hline Reliability and maintenance & Low & High & High \\
\hline Manufacturing costs & High & Low & Medium \\
\hline
\end{tabular}

As a result of the analyses, it was found that each version presents its advantages and disadvantages, but the most reliable seems to be the third one, especially due to its simple construction and due to the ease of manufacturing point of view, as well as how expensive they are and how difficult is to assemble the manufactured parts together, in order to build the assembly at the end. Another decisive feature of the selected version was its efficiency in operation, which is related basically to the feeding accuracy of the extruder. Further analysis has been made for version 3 , determining that the main weak point of the assembly design is related to the base plate, presented in Figure 2 a) and b), which is mounted directly on the stepper motor, on which the whole assembly is attached.

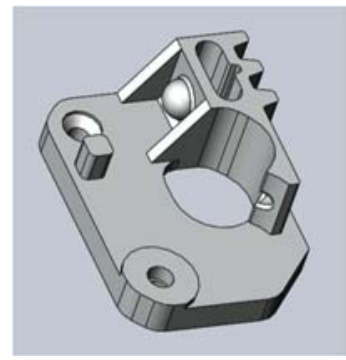

a

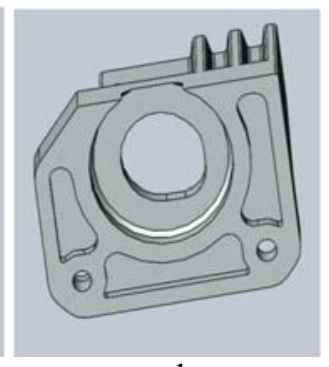

b

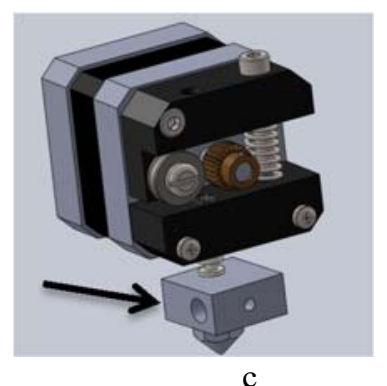

c

Fig. 2. The main component of the selected constructive version for the extrusion system. 
As it can be noticed from Figure 2 a) and b), instead of using one main plate with high complexity and manufacturing costs, it was decided to use the plate as shown in Figure $2 \mathrm{c}$ ), having much simpler geometry, having the same role in the assembly as compared to the previous one. Because of the simple geometry of the newly designed plate, this could be easily manufactured by using a CNC milling center, in a short amount of time, so its cost is insignificant as compared to the cost of the original part.

For validating the design, last, but not least, finite element analysis method has been considered to be realized with SolidWorks Simulation module. A critical aspect of the analysis is the intensity of the thermal demands to which the assembly is subjected. These demands are imposed by the thermal characteristics of the extruded material. The material considered as raw material for this research was $\mathrm{Sn} 60 \mathrm{~Pb} 40$ in the form of a filament with a diameter of $1.5 \mathrm{~mm}$. The optimum extrusion temperature of this type of material varies slightly around the temperature of $188^{\circ} \mathrm{C}$.

As it can be seen from Figure 3 a), the assembly considered for analysis has a symmetrical construction on the $\mathrm{Y}$ axis, which is the main reason why a symmetrical analysis has been performed, with respects to the symmetry plane shown in Figure 3 a). For performing the simulation as shown in Figure $3 \mathrm{~b}$ ), the assembly file containing all the constructive components of the sub-assembly extrusion system was opened in SolidWorks simulation program, with ".sldasm" extension (SolidWorks assembly file). Thermal type of finite element analysis has been further on selected.

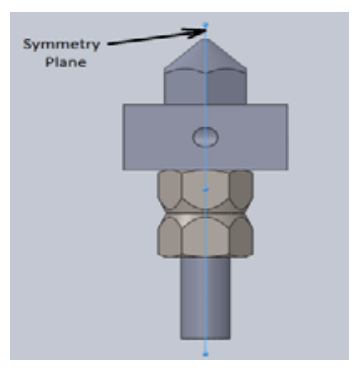

a

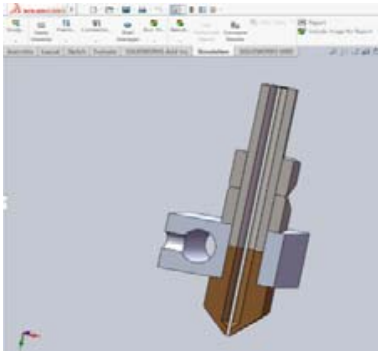

b

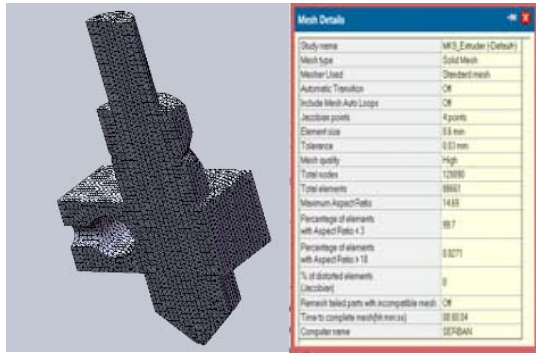

d

Fig. 3. CAD model considered for the thermal FEA analysis.

The raw material was not available in the SolidWorks Simulation material library, so the material characteristics were defined as they were found in the literature [11]. The Sn60Pb40 tin-lead alloy is common in electronics, being regarded as the most popular leaded alloy for dipping. It has low cost and good bonding properties. It rapidly dissolves gold and silver, so it is not recommended to be used in combinations with such materials. Slightly cheaper than $\mathrm{Sn} 63 \mathrm{~Pb} 37$, this type of material is very often used due to the cost reasons as the melting point difference is insignificant in practice. On slow cooling, these materials give slightly duller joints than Sn63Pb37. Figure $3 \mathrm{c}$ ) shows the meshing of the 3D model in the SolidWorks Simulation program. A dense mesh has been considered for the analysis, with the total number of tetrahedral elements being 128890 , and the total number of nodes being 886611 , as shown in Figure $3 \mathrm{~d}$ ).

The constraints and demands to which the analyzed model is subjected were introduced at this stage of the analysis, by taking into account the operating principle of the subassembly, namely the classic extrusion and the fact that it must be taken into account that the thermal stresses to which the mechanism is subjected are due to convection heating. This aspect is realized by using an electrical resistance. In the case of the current made simulation, the electric resistance influence and effect was neglected in order to simplify the made analysis. 


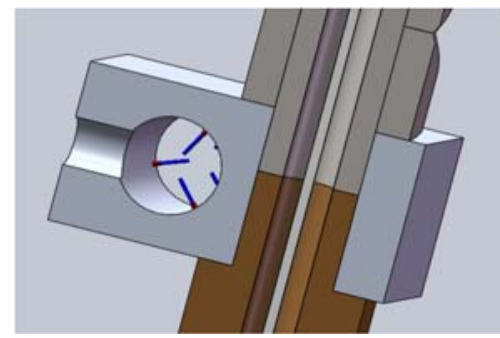

$\mathrm{a}$

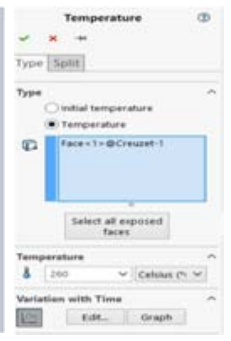

$\mathrm{b}$

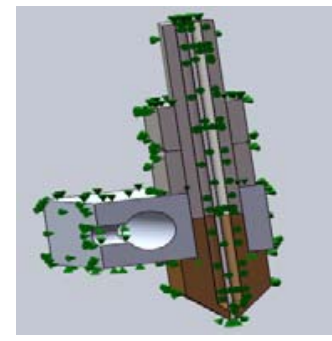

c

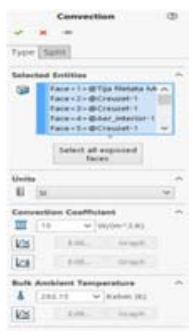

d

Fig. 4. Constrains imposed for the finite element analysis.

In the case of the Finite Element Analysis made, the heating of the contact area with the electrical resistance of the crucible was replaced by applying the "temperature" constraint with a temperature applied to the surface in such way that it will not adversely affect the results obtained. The surface on which this constraint was applied materializes the same thermal effect as when the electrical resistance is used. In Fig 4 a) is presented this surface, as a representative image where the temperature constraint has been applied (see Figure $4 \mathrm{~b}$ ). The selected temperature was $260^{\circ} \mathrm{C}$, this temperature being the maximum temperature that the $3 \mathrm{D}$ printer could provide. In order to have the simulation as closed as possible to the real behavior of the sub-assembly system, convection constraints have been applied to the surfaces in contact with the environment, as shown in Figure $4 \mathrm{c}$ ) and d). In other words, all the external surfaces were assumed to exchange heat (or not) with the environment. In the made analysis, the environment has been considered as having $20^{\circ} \mathrm{C}$ temperature in normal conditions $(293.15 \mathrm{~K})$. Also, the convection coefficient of 15 [W/ $2 * \mathrm{~K}]$ was set, in this case. This value of the convection coefficient was considered as being the mean value in the case of free convection through gases and dried vapors, because the phenomenon that occurs in this case is a free convection through air, which is a mixture of gases, so it can be considered a gas.

\section{Results and conclusions}

Most of the 3D printers on the market are using electrical resistance for heating the hot-end, the most common ones being the $40 \mathrm{~W}$ cylindrical resistors with diameter of $6 \mathrm{~mm}$ and length of $20 \mathrm{~mm}$. Because these resistors are most common in practice, it was selected such a resistance for heating the hot-end of the sub-assembly. During the simulation, the distribution of temperatures throughout the assembly was monitored in order to highlight the efficiency of the heating element. In Figure5 there are presented the results obtained after the thermal simulation run. From Figure 5 a) it can be seen that the heat distribution is varying, the resulted temperatures being between 135 and $260^{\circ} \mathrm{C}$. The way the temperatures are distributed materializes the ideal heat transfer pattern to the extrusion nozzle. A negligible aspect is the functional role of the electrical resistance support and the extrusion nozzle. This component aims to homogenize the temperatures in the heating zone. The heat produced by the source is uniformly absorbed. A disadvantage of the first constructive version, as one may notice from the image presented in Figure $5 \mathrm{a}$ ) is the fact that the heat is transmitted to the supporting plate of the stepper motor, so in order to avoid any malfunctions of the motor, a fan will be needed to be used in order to cool it down during the 3D printing process. For this analysis, the material for the crucible was considered aluminum and the material for the nozzle was considered brass. Due to the fact that aluminum and brass are more expensive materials as compared to plain carbon steel, in order to decide whether these two components will be made of aluminum/brass or plain carbon steel, another FEA analysis was performed in the same conditions as the first one. 


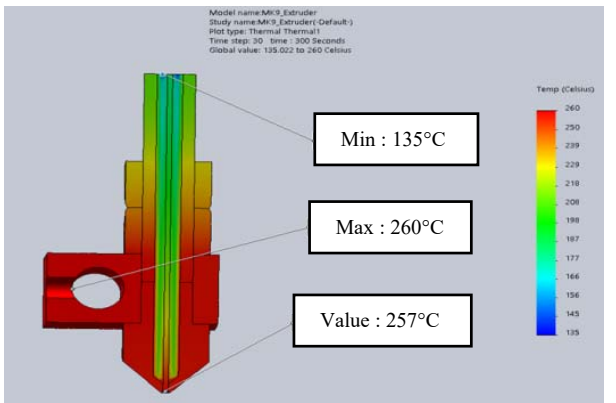

$\mathrm{a}$

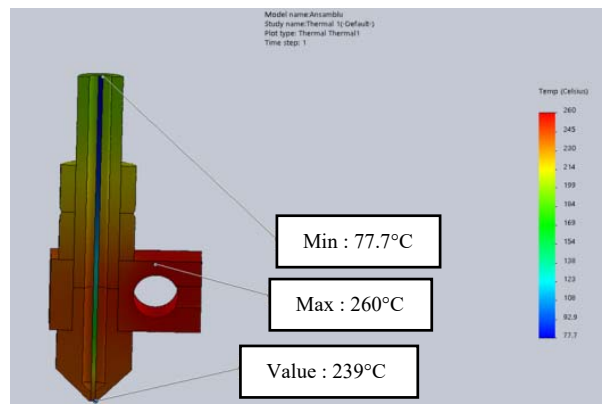

b

Fig. 5. Variation of the temperature within the hot-end made out of aluminum, brass and steel / plain carbon steel.

While performing the second analysis, all the initial parameters and conditions were kept the same as in the case of the first one, except the materials of the crucible and the nozzle. The material characteristics for the nozzle and crucible were loaded from the SolidWorks library program. When comparing the results of the second finite element analysis with the results of the first one, it can be easily noticed that the temperature distribution is much better in the first case presented in Figure $5 \mathrm{a}$ ). The temperature difference between the hottest point and the tip of the nozzle is just $3{ }^{\circ} \mathrm{C}$ in this case. In the second case presented in Figure $5 \mathrm{~b}$ ), the temperature in the tip of the nozzle was much lower than in the hottest point of the hotend, the difference of temperatures between these elements being in this case approximately $21^{\circ} \mathrm{C}$. In conclusion, in terms of material choice, after performing the second analysis, it was decided that the best option is the combination of aluminum, brass and steel for the manufacturing of the hot-end of the sub-assembly extrusion system, rather than manufacturing it entirely from plain carbon steel. The decisive factor for making this decision was the fact that the thermal conductivity of the aluminum and brass is much better and the fact that the parts are very small, the difference in term of costs between the two analyzed versions, being insignificant in this case. The extrusion system was finally manufactured and tested on a commercial 3D desktop printer, Prusa i3 type that is available at the Technical University of Cluj-Napoca (TUCN).

\section{References}

1. C.S. Miron Borzan, M.C. Dudescu, V. Ceclan, A. Trif, M. Ridzon, P. Berce, Materiale Plastice, 53, 1, (2016)

2. M. Ulmeanu, C. Doicin, D. Baila, A. Rennie, C. Neagu, S. Lahan, Materiale Plastice, 52, 3, (2015)

3. C. Cosma, N. Balc, M. Moldovan, L. Morovic, P. Gogola, C.S. Miron-Borzan, Journal of Optoelectronics and Advanced Materials, 19, 11-12, (2017)

4. D. Kazmer, Injection Mold Design Engineering”, Hanser Publishers, (2016)

5. A. Luca, N. Balc, A. Popan, M. Balas, Annals Of Oradea University, Fascicle of Management and Technological Engineering, 19, 9, (2010)

6. S. Masood, Journal of Materials \& Design, 25, 7, (2004)

7. A. Equbal, Procedia Materials Science, 6, (2014)

8. C. R. Garcia, Prog. Electromagn. Res. Lett., 34, (2012)

9. J. Mireles, Journal of Electronic Packaging, 2012

10. C. Lieberwirth, A. Harder, H. Seitz, Journal of Mechanics Engineering and Automation, 7 (2017)

11. MatWeb - Material Property Data - http://www.matweb.com 